\title{
The Uncertainty Principle and Hypoelliptic Operators
}

\author{
By \\ Yoshinori MORIMOTO*
}

\section{Introduction}

Let $P$ be a differential operator of second order with coefficients in $C^{\infty}\left(R^{n}\right)$, that is,

$$
P=\sum_{j, k} a_{j k}(x) D_{j} D_{k}+\sum_{j} i b_{\jmath}(x) D_{j}+c(x), D_{j}=-i \partial_{x_{j}}
$$

We assume that

$$
\left\{\begin{array}{l}
a_{j k} \text { and } b_{j} \text { are real valued, } \\
\sum a_{j k}(x) \xi, \xi_{k} \geq 0 \quad \text { for any }(x, \xi) \in R^{2 n} .
\end{array}\right.
$$

Let $\log \Lambda$ be a pseudodifferential operator with symbol $\log \langle\xi\rangle$. As a criterion for $P$ to be hypoelliptic in $R^{n}$ the author has given the following in [8] (see also [6]) :

Theorem A. (Corollary 2 in [8]) If for any $\varepsilon>0$ and any compact set $K$ of $R^{n}$ there exists a constant $C_{\varepsilon, K}$ such that

$$
\|(\log \Lambda) u\|^{2} \leq \varepsilon \operatorname{Re}(P u, u)+C_{\varepsilon, K}\|u\|^{2}, \quad u \in C_{0}^{\infty}(K),
$$

then $P$ is hypoelliptic in $R^{n}$. Furthermore, we have

$$
\text { WF } P v=W F \quad \text { for } v \in \mathscr{D}^{\prime}\left(R^{n}\right) \text {. }
$$

Though more general criteria than Theorem A were given in [8] and [9], in the present paper we shall restrict our consideration to the application of this theorem to some class of degenerate elliptic operators of second order. To verify the estimate (3), it is convenient

Communicated by S. Matsuura, September 13, 1986.

* Department of Engineering Mathematics, Faculty of Engineering, Nagoya University, Nagoya 464, Japan.

Current address: Institute of Mathematics, Yoshida College, Kyoto University, Kyoto 606, Japan. 
to employ "the uncertainty principle" mathematically formulated by Fefferman-Phong [2] and Fefferman [1].

This paper consists of two sections. In Section 1, we slightly modify the result in [2, Section 2] (, see Theorem B in Section 1). In Section 2 we study the hypoellipticity of infinitely degenerate elliptic operators by using Theorems A and B.

We mention that only the simplest parts of arguments in [1] and [2] are used here. One might expect to characterize the operators satisfying (3) by means of much deeper arguments in [1] and [2].

The author wishes to thank gratefully Prof. T. Matsuzawa for valuable discussions.

\section{§1. The Uncertainty Principle}

Let $\lambda$ be $0<\lambda \leq 1$. We consider a symbol of the form $a(x, \xi)=$ $|\xi|^{2 \lambda}+V(x), x \in R^{n}$, where $V(x)$ is a non-negative measurable function and depends on a large parameter $M>0$, that is, $V(x)=V(x$, $M$ ). (In the next section we shall employ the case when $V(x)=g(x)$ $M^{2}, g \in C^{\infty}$.)

Let $R \equiv R(M)$ be a non-decreasing function of $M$ such that $R \rightarrow$ $\infty$ as $M \rightarrow \infty$. Let $\mathscr{C}$ denote a set of boxes

$$
B \equiv\left\{(x, \xi) \in R^{2 n} ;\left|x_{j}-x_{0 j}\right| \leq \delta / 2,\left|\xi_{j}-\xi_{0 j}\right| \leq \delta^{-1} / 2\right\}
$$

for all $\left(x_{0}, \xi_{0}\right) \in R^{2 n}$ and all $\delta>0$. Clearly, the volume of $B \in \mathscr{C}$ is equal to 1 .

Theorem B. Assume that there exists a $c>0$ such that for any $B \in \mathscr{C}$

$$
m(\{(x, \xi) \in B ; a(x, \xi) \geq R\}) \geq c,
$$

where $m($.$) denotes Lebesgue measure. Then we have$

$$
\left\|\left|D_{x}\right|^{2} u\right\|^{2}+(V(x) u, u) \geq c^{\prime} R\|u\|^{2}, u \in C_{0}^{\infty}\left(R^{n}\right),
$$

where $c^{\prime}>0$ depends only on $c$ and $n$.

For the comparison of Theorem B with the original version in [2, Section 2] we set the following corollary though we shall not use it throughout the present paper.

Corollary. Let $V(x)$ belong to $C^{\infty}\left(R_{x}^{n}\right)$ and satisfy 


$$
\left|D^{\alpha} V(x)\right| \leq C_{\alpha} M^{2}, \quad x \in R^{n} \text {. }
$$

Assume that for some $\varepsilon>0$ and $c>0$ we have $R \geq c M^{\varepsilon}$. Then the estimate

(7) still holds when the hypothesis (6) is replaced by

$$
\min _{B \in \mathscr{C}} \max _{(x, \xi) \in B} a(x, \xi) \geq R \text { 。 }
$$

For the proof of Theorem $B$ in the case $\lambda=1$ we use the following lemma given by Fefferman [1]:

Lemma 1. Assume that $V(x) \geq 0$, measurable on a cube $Q$ in $R^{n}$. Suppose that there exists a $c>0$ such that

$$
m\left(\left\{x \in Q ; V(x) \geq c(\operatorname{diam} Q)^{-2}\right\}\right) \geq c|Q| .
$$

Then for $u \in C^{1}$ we have

$$
\int_{Q}\left\{|\nabla u(x)|^{2}+V(x)|u(x)|^{2}\right\} d x \geq c^{\prime}(\operatorname{diam} Q)^{-2} \int_{Q}|u(x)|^{2} d x .
$$

The constant $c^{\prime}>0$ depends only on $n$ and $c$.

In the Main Lemma of $\left[1, p_{0} 146\right]$, it is assumed that $V(x)$ is polynomial and $\left(\operatorname{Av}_{Q} V\right) \geq(\operatorname{diam} Q)^{-2}$. The proof in [1] is still valid under the above hypotheses.

For the case $0<\lambda<1$ we need the following two lemmas: The first is a modification of Lemma 1.

Lemma 2. Assume that $V(x) \geq 0$, measurable on a cube $Q$ in $R^{n}$. For a $0<\lambda<1$, suppose that there exists a $c>0$ such that

$$
m\left(\left\{x \in Q ; V(x) \geq c(\operatorname{diam} Q)^{-2 \lambda}\right\}\right) \geq c|Q| .
$$

Then for $u \in C^{1}$ we have

$$
\begin{aligned}
& \int_{Q \times Q}\left\{|u(x)-u(y)|^{2} /|x-v|^{n+2 \lambda}\right\} d x d y \\
& \quad+\int_{Q} V(x)|u(x)|^{2} d x \geq c^{\prime}(\operatorname{diam} Q)^{-2 \lambda} \int_{Q}|u(x)|^{2} d x .
\end{aligned}
$$

Proof of Lemma 2. Note that

$$
\begin{aligned}
& \int_{Q \times Q}\left\{|u(x)-u(y)|^{2} /|x-y|^{n+2 \lambda}\right\} d x d y \\
& \geq c \frac{(\operatorname{diam} Q)^{-2 \lambda}}{|Q|} \int_{Q \times Q}|u(x)-u(y)|^{2} d x d y,
\end{aligned}
$$


where $c$ depends only on $n$. Using this formula instead of the similar one in the proof of the Main Lemma of [1], we can obtain the desired estimate. The detail is omitted.

Q.E.D

Lemma 3. For $0<\lambda<1$ we have

$$
\begin{aligned}
& \left\|\left|D_{x}\right|^{\lambda} u\right\|^{2} \\
& \quad=c \int_{R^{n} \times R^{n}}\left\{|u(x)-u(y)|^{2} /|x-y|^{n+2 \lambda}\right\} d x d y, u \in C_{0}^{\infty}\left(R^{n}\right),
\end{aligned}
$$

where $c$ depends only on $\lambda$ and $n$. (See [11]).

Proof of Theorem B. Let $Q_{x_{0}}$ be a cube in $R_{x}^{n}$, that is,

$$
Q_{x_{0}}=\left\{x \in R^{n} ;\left|x_{j}-x_{0 j}\right| \leq \delta / 2\right\} \quad \text { for some } x_{0} \in R^{n}, \delta>0 .
$$

If $\delta=2^{-2} n^{1 / 2}(2 / R)^{1 / 2 \lambda}$ it follows from (6) that

$$
m\left(\left\{x \in Q_{x_{0}} ; V(x) \geq R / 2\right\}\right) \geq c\left|Q_{x_{0}}\right| .
$$

In fact, this is obvious if we consider a box $Q_{x_{0}} \times\left\{\xi ;\left|\xi_{j}\right| \leq n^{-1 / 2}\right.$ $\left.(R / 2)^{1 / 2 \lambda}\right\}$ belonging to $\mathscr{C}$. Let $\left\{Q_{j}\right\}$ be a partition of $R_{x}^{n}$ such that each $Q_{j}$ is a translation of $Q_{x_{0}}$ with $\delta=2^{-2} n^{1 / 2}(2 / R)^{1 / 2 \lambda}$. Note diam $Q_{j}=2^{-1} n^{1 / 2}(2 / R)^{1 / 2 \lambda}$. When $\lambda=1$, in view of (13) we have (7) by Lemma 1 because $\left\|\left|D_{x}\right| u\right\|^{2}=\|\nabla u\|^{2}$. Note that

$$
\int_{R^{n} \times R^{n}} d x d y \geq \sum_{j} \int_{Q_{j} \times Q_{j}} d x d y .
$$

Then, the estimate (7) for the case $0<\lambda<1$ also follows from Lemmas 2 and 3.

Q.E.D.

Proof of Corollary. It follows from (9) that we have

$$
\max _{Q_{x_{0}}} V(x) \geq R / 2
$$

for any $Q_{x_{0}}$ defined by (12) with $\delta=2^{-2} n^{1 / 2}(2 / R)^{1 / 2 \lambda}\left(\equiv \delta_{0}\right)$. Setting $F(y)=V\left(\delta_{0} y+x_{0}\right)$, by (8) we have

$$
\left|D_{y}^{\alpha} F(y)\right| \leq C_{\alpha}^{\prime} M^{2} R^{-|\alpha| / 2 \lambda} \leq C_{\alpha}^{\prime \prime} M^{2-\varepsilon|\alpha| / 2 \lambda}, y \in R^{n} .
$$

Fix a large integer $N$ such that $\varepsilon N \geq 4 \lambda$ and set

$$
P(y)=\sum_{|\alpha|<N} \partial_{y}^{\alpha} F(0) y^{\alpha} / \alpha ! \text {. }
$$

Then for some $C_{0}$ independent of $M$ we have 


$$
\begin{aligned}
& |F(y)-P(y)| \leq C_{0} \\
& \quad \text { on a unit cube } Q_{0} \equiv\left\{y ;\left|y_{j}\right| \leq 1 / 2\right\} .
\end{aligned}
$$

By (14) and (15) we have $\max _{Q_{0}} P \geq R / 4$ when $M$ is large enough. Since $P$ is polynomial of order $N$, we have $\max _{Q_{0}}|\nabla P| \leq C \max _{Q_{0}}|P|$, where $C$ depends only on $N$ and $n$. In view of (15) and $F \geq 0$ we have $\max _{Q_{0}} P=\max _{Q_{0}}|P|$ if $M$ is large enough. If $P\left(y_{0}\right)=\max _{Q_{0}} P$ and if $Q_{0}^{\prime}$ is a subcube of $Q_{0}$ such that $y_{0} \in Q_{0}^{\prime}$, then for $y \in Q_{0}^{\prime}$ we have

$$
\begin{aligned}
P(y) & \geq P\left(y_{0}\right)-\max _{Q_{0}^{\prime}}|\nabla P|\left|y-y_{0}\right| \\
& \geq P\left(y_{0}\right)-\max _{Q_{0}}|\nabla P|\left|y-y_{0}\right| \\
& \geq P\left(y_{0}\right)\left(1-C\left|y-y_{0}\right|\right) .
\end{aligned}
$$

Hence, there exists a subcube $Q_{0}^{\prime}$ of $Q_{0}$ such that $P(y) \geq R / 8$ on $Q_{0}^{\prime}$ and $\left|Q_{0}^{\prime}\right| \geq c_{0}$ for a constant $c_{0}$ depending only on $n$ and $N$. Using (15) again, we have $V(x) \geq R / 16$ on some subcube $Q_{x}^{\prime}$ of $Q_{x}$ with $\left|Q_{x}^{\prime}\right| /\left|Q_{x}\right| \geq c_{0}$ if $M$ is large enough. So, for any $Q_{x}$ defined by (12) with $\delta=\delta_{0}$ we have the similar formula as (13). Q.E.D.

\section{§ 2. Hypoelliptic Operators}

Let $P_{0}=p_{0}\left(x, y, D_{x}\right)$ be a differential operator of second order with $C^{\infty}$ coefficients of the form

$$
P_{0}=a\left(x, y, D_{x}\right)+g\left(x^{\prime}\right) b\left(x, y, D_{y}\right) \text { in } R^{n}=R_{x}^{n_{1}} \times R_{y}^{n_{2}},
$$

where $x=\left(x^{\prime}, x^{\prime \prime}\right) \in R_{x^{\prime}}^{n_{1}^{\prime}} \times R_{x^{\prime \prime}}^{n_{1}^{\prime \prime}}$. We assume that $a\left(x, y, D_{x}\right)$ and $b\left(x, y, D_{y}\right)$ are strongly elliptic with respect to $x$ and $y$, respectively, that is,

$$
\text { (A-1) } \quad \begin{cases}\operatorname{Re} a(x, y, \xi) \geq c_{1}|\xi|^{2} & \text { for }(x, y) \in R^{n} \text { and large }|\xi|, \\ \operatorname{Re} b(x, y, \eta) \geq c_{2}|\eta|^{2} & \text { for }(x, y) \in R^{n} \text { and large }|\eta|,\end{cases}
$$

where $c_{1}$ and $c_{2}$ are positive constants. Furthermore we assume

$$
\left\{\begin{array}{l}
g^{(j)}(0)=0 \text { for any integer } j \geq 0 \\
\text { and } g\left(x^{\prime}\right)>0 \text { for } x^{\prime} \neq 0
\end{array}\right.
$$

The conjunction of Theorems $\mathrm{A}$ and $\mathrm{B}$ gives the following:

Proposition 1. Let $P_{0}$ be the above operator and assume $(A-1)$ and 
(A-2). Furthermore, assume that $g\left(x^{\prime}\right)$ satisfies

$$
\lim _{x^{\prime} \rightarrow 0}\left|x^{\prime}\right|\left|\log g\left(x^{\prime}\right)\right|=0 \text {. }
$$

Then $P_{0}$ is hypoelliptic in $R^{n}$.

Remark 1. The theorem is nothing but Theorem 1.1 of [7], where we employed a primitive method in place of Theorems A and $B$.

Remark 2. As a typical example of $P_{0}$ we have $D_{x_{1}}^{2}+D_{x_{2}}^{2}+g\left(x_{1}\right) D_{y}^{2}$ in $R^{3}$. The theorem for this example under a little stringent assumption on $g$ was proved by Kusuoka-Strook by using the Malliavin calculus (see Theorem 8.41 of [4]). For this example, it was proved in [4] and [5] that if $g\left(x_{1}\right)=g\left(-x_{1}\right)$ and $g$ is nondecreasing in $R_{+}$ then (17) is necessary for the hypoellipticity.

Remark 3. If $g=\exp \left(-1 /\left|x^{\prime}\right|^{\sigma}\right), \sigma>0$, then (17) means $\sigma<1$.

Proof of Proposition 1. By Theorem A it suffices to derive (3) for $P_{0}$. Since our consideration is local on a fixed compact set $K$, by modifying $g$ outside of $K$ we may assume $g\left(x^{\prime}\right) \geq c_{0}>0$ for $\left|x^{\prime}\right| \geq 1$. It follows from (A-1) that for some constant $C$ we have

$$
\begin{aligned}
& C\left(\operatorname{Re}\left(P_{0} u, u\right)+\|u\|^{2}\right) \\
& \quad \leq\left\|\nabla_{x^{\prime}} \check{u}\right\|^{2}+\left\|\nabla_{x^{\prime \prime}} \check{u}\right\|^{2}+\left(g\left(x^{\prime}\right)|\eta|^{2} \check{u}, \check{u}\right), u \in C_{0}^{\infty},
\end{aligned}
$$

where $\ddot{u}=\check{u}(x, \eta)$ is the Fourier transform of $u$ with respect to $y$. Set $V\left(x^{\prime}\right)=g\left(x^{\prime}\right)|\eta|^{2}$. We shall show that for any integer $k>0$ there exists a $M_{k}>0$ such that

$$
\begin{aligned}
\int\left\{\mid \nabla_{x^{\prime}} u\left(x^{\prime}\right)\right. & \left.\left.\right|^{2}+V\left(x^{\prime}\right)\left|u\left(x^{\prime}\right)\right|^{2}\right\} d x^{\prime} \\
& \geq c(k \log |\eta|)^{2} \int\left|u\left(x^{\prime}\right)\right|^{2} d x^{\prime} \\
& \text { for } u \in C_{0}^{\infty}\left(R_{x^{\prime}}^{n_{1}}\right) \quad \text { if }|\eta| \geq M_{k},
\end{aligned}
$$

where $c>0$ is a constant independent of $k$ and $|\eta|$. The estimate (3) easily follows from (19). Instead of $x^{\prime}$ and $|\eta|$, write $x$ and $M$. Use Theorem B with $\lambda=1$. We shall check (6). In view of (17), for any $k$ there exists a $\tau_{k}>0$ such that 
(20)

$$
-\log g(x)=|\log g(x)| \leq 1 /\left(10 k(|x|) \quad \text { for }|x| \leq \tau_{k} .\right.
$$

Take a large $M_{k}>0$ such that

$$
M_{k}^{-1} \leq \min _{|x| \geq \tau_{k}} g(x) \text { and } \tau_{k} \geq\left(10 k \log M_{k}\right)^{-1} .
$$

When $M \geq M_{k}$ we have

$$
V(x) \geq M \quad \text { for }|x| \geq \tau_{k} .
$$

Set $R \equiv R(M)=(k \log M)^{2}$ and note that

$$
\begin{aligned}
V(x) & =M \exp \{\log M-|\log g(x)|\} \\
& \geq M \exp \{\log M-1 /(10 k|x|)\} \geq M \\
& \text { if }(10 R)^{-1 / 2} \leq|x| \leq \tau_{k} \text { and } M \geq M_{k} .
\end{aligned}
$$

Inequalities (21) and (22) show that for $Q_{x_{0}}$ defined by (12) with $\delta \geq(8 R)^{-1 / 2}$ we have

$$
m\left(\left\{x \in Q_{x_{0}} ; V(x) \geq M\right\}\right) \geq\left|Q_{x_{0}}\right| / 10 .
$$

From this we see that (6) holds for $B \in \mathscr{C}$ with $\delta \geq(8 R)^{-1 / 2}$. On account of the term $|\xi|^{2},(6)$ is trivial for others $B \in \mathscr{C}$.

Q.E.D.

The above discussion can apply to more degenerate elliptic operators than $P_{0}$. For example:

Corollary. Proposition 1 is still true when $g\left(x^{\prime}\right)$ is replaced by $g\left(x^{\prime}\right)$ $\sin ^{2}\left(1 /\left|x^{\prime}\right|\right)$ 。

Proof. As in the proof of Proposition 1, we write $x$ and $M$ instead of $x^{\prime}$ and $|\eta|$. Let $Q_{x_{0}}$ be a cube defined by (12). For any $Q_{x_{0}}$ where $\sin (1 /|x|)$ vanishes at least with two different values of $|x|$, we have

$$
m\left(\left\{x \in Q_{x_{0}} ; \sin ^{2}(1 /|x|) \geq 1 / 2\right\}\right) \geq\left|Q_{x_{0}}\right| / 4 .
$$

For others $Q_{x_{0}}$ in $\{|x| \leq T\}$ with $\delta \geq(8 R)^{-1 / 2}$ we have

$$
m\left(\left\{x \in Q_{x_{0}} ; \sin ^{2}(1 /|x|) \geq c_{n} T^{-4} R^{-1}\right\}\right) \geq\left|Q_{x_{0}}\right| / 2
$$

because $\sin t \geq 2 t / \pi$ for $0 \leq t \leq \pi / 2$. Here $c_{n}>0$ depends only on $n$. Noting (23), from (24) or (25) we see that (6) holds even when $V(x)=g(x) \sin ^{2}(1 /|x|) M^{2}$.

Q.E.D. 
We can give a more pathological hypoelliptic operator. Let $P_{1}$ be a second order differential operator of the form (16) with $g\left(x^{\prime}\right)=$ $f\left(\left|x^{\prime}\right|\right)$ for $f \in C^{\infty}\left(\bar{R}_{+}\right)$. We assume that $f(t)$ vanishes on a Cantor set $E$ with measure 0 defined as follows : Set $E=I_{0} \backslash \bigcup_{j=1}^{\infty} I_{j}$, where $I_{0}=$ $[0,1], I_{1}$ is an open interval with length $1 / 3$ whose center is $1 / 2$. Here $I_{2}$ and $I_{3}$ are open intervals with length $(1 / 3)^{2}$ whose centers coincide with the centers of each connected component of $I_{0} \backslash I_{1}$, respectively. Furthermore, $I_{4}, \ldots, I_{7}$ are open intervals with lenghth $(1 / 3)^{3}$ whose centers coincide with the centers of each connected component of $I_{0} \mid \bigcup_{j=1}^{3} I_{j}$, respectively. We define open intervals $I_{j}$ in this manner, recursively. On each $I_{j}=(-a+b, a+b), j \geq 1$, we set $f(t)=\exp \left\{\left(-1 /|t+a-b|^{\sigma}\right)+\left(-1 /|t-a-b|^{\sigma}\right)\right\}$ and we set $\exp (-1 /$ $\left(|t-1|^{\circ}\right)$ for $(1, \infty)$, where $\sigma>0$.

Proposition 2. Let $P_{1}$ be a second order differential operator of the form (16) with $g\left(x^{\prime}\right)=f\left(\left|x^{\prime}\right|\right)$ for $f \in C^{\infty}\left(\bar{R}_{+}\right)$defined above. We assume $(A-1)$. If $0<\sigma<1$ then $P_{1}$ is hypoelliptic in $R^{n}$.

Proof. Recall Remark 3 of Proposition 1, that is, that the condition (17) is equivalent to $\sigma<1$ when $g\left(x^{\prime}\right)=\exp \left(-1 /\left|x^{\prime}\right|^{\sigma}\right)$. Noting the definition of the Cantor set $E$ we can easily check the condition (6) of Theorem B, as in the proof of Proposition 1. The detail is omitted.

Q.E.D.

The assumption (A-1) can be also weakened. Assume that

$$
a\left(x, y, D_{x}\right)=-\sum_{j=1}^{r} X_{j}^{2} \text { and } b\left(x, y, D_{y}\right)=-\sum_{j=1}^{s} Y_{j}^{2},
$$

where $X_{j}\left(\operatorname{resp} . Y_{j}\right)$ are real vector fields in $R_{x}^{n_{1}}\left(\right.$ resp. $\left.R_{y}^{n_{2}}\right)$ for every fixed $y \in R_{y}^{n_{2}}$ (resp. $\left.x \in R_{x}^{n_{1}}\right)$. In place of (A-1) we assume that $(\mathrm{A}-1)^{\prime}\left\{\begin{array}{l}\text { For any fixed } y \in R_{y}^{n_{2}}\left(\text { resp. } x \in R_{x}^{n_{1}}\right) \text { the vector fields } X_{1}, \ldots, \\ X_{r}\left(\text { resp. } Y_{1}, \ldots, Y_{s}\right) \text { and their repeated commutators span the } \\ \text { tangent space at each point in } R_{x}^{n_{1}}\left(\text { resp. } R_{y}^{n_{2}}\right) .\end{array}\right.$ and 
(B) $\left\{\begin{array}{l}\text { For any fixed } y \in R_{y}^{n_{2}} \text { the vector fields } X_{1}, \ldots, X_{r} \text { and their } \\ \text { repeated commutators of order up to } l \text { span the subtangent } \\ \text { space } T\left(R_{x^{\prime}}^{n^{\prime}}\right) \text { at each point in } R_{x}^{n_{1}} .\end{array}\right.$

Proposition 3. Let $P_{2}$ be a differential operator of the form(16). Assume that $(A-1)^{\prime},(A-2)$ and $(B)$. If $g\left(x^{\prime}\right)$ satisfies

$$
\lim _{x^{\prime} \rightarrow 0}\left|x^{\prime}\right|^{1 /(l+1)}\left|\log g\left(x^{\prime}\right)\right|=0 \text {. }
$$

then $P_{2}$ is hypoelliptic in $R^{n}$.

Proof. It follows from $(\mathrm{A}-1)^{\prime}$ and (B) that for a $k>0$ we have

$$
\begin{aligned}
& C\left(\operatorname{Re}\left(P_{2} u, u\right)+\|u\|^{2}\right) \\
& \geq\left\|<D_{x}>^{k} \check{u}\right\|^{2}+\left\|\left|D_{x^{\prime}}\right|^{1(l+1)} \check{u}\right\|^{2}+\left(g\left(x^{\prime}\right)|\eta|^{2 k} \check{u}, \check{u}\right), u \in C_{0}^{\infty},
\end{aligned}
$$

instead of (18). Indeed, this is a direct consequence of Hörmander's classical theorem in [3] and its sharp version given in [10] and [2]. In place of $x^{\prime}$ and $|\eta|^{k}$, write $x$ and $M$. Set $V(x)=g(x) M^{2}$. Then the proof of the proposition is reduced to show that for any integer $k>0$ there exists a $M_{k}>0$ such that

$$
\begin{aligned}
& \left\|\left|D_{x}\right|^{1 /(l+1)} u\right\|^{2}+(V(x) u, u) \\
& \quad \geq c(k \log M)^{2}\|u\|^{2}, \quad u \in C_{0}^{\infty}, \\
& \text { if } M \geq M_{k} .
\end{aligned}
$$

For the proof of (28) it suffices to apply Theorem B with $\lambda=1$ / $(l+1)$. Similarly as in the proof of Proposition 1, the condition (6) easily follows from (26).

Q.E.D.

\section{References}

[1] Fefferman C., The uncertainty principle, Bull. Amer. Math. Soc., 9 (1983), 129-206.

[2] Fefferman C. and Phong D.H., The uncertainty principle and sharp Gårding inequalities, Comm. Pure Appl. Math., 34 (1981), 285-331.

[3] Hörmander L., Hypoelliptic second order differential equations, Acta Math., 119 (1967), 147-171.

[4] Kusuoka K. and Strook D., Applications of the Malliavin calculus, Part II, J. Fac. Sci. Univ. Tokyo Sect. IA, Math., 32 (1985), 1-76.

[5] Morimoto Y., Non-hypoellipticity for degenerate elliptic operators, Publ. RIMS Kyoto Univ., 22 (1986), 25-30.

[6] - On a criterion for hypoellipticity, Proc. Japan Acad., 62 A (1986), 137-140.

[7] Hypoellipticity for infinitely degenerate elliptic operators, Osaka J.Math., 
24-1 (1987), 13-35.

[8] Morimoto Y., A criterion for hypoellipticity of second order differential operators, Osaka J. Math., 24-3 (1987), 651-675.

[9] Criteria for hypoellipticity of differential operators, Publ. RIMS Kyoto Univ., 22 (1986), 1129-1154.

[10] Rothchild L. and Stein E. M., Hypoelliptic differential operators and nilpotent groups, Acta Math., 137 (1976), 247-320.

[11] Slobodeskii L. N., Generalized spaces of S. L. Sobolev and their application to boundary value problems for partial differential equations, Uch. Zap. Lenin. Gos. Univ., 197 (1958), 54-112. 